\title{
Equilibrium measures on trees
}

\author{
Nicola Arcozzi ${ }^{1} \cdot$ Matteo Levi ${ }^{2}$ (I)
}

Received: 19 November 2020 / Accepted: 11 September 2021 / Published online: 23 September 2021

(c) The Author(s) 2021

\begin{abstract}
We give a characterization of equilibrium measures for $p$-capacities on the boundary of an infinite tree of arbitrary (finite) local degree. For $p=2$, this provides, in the special case of trees, a converse to a theorem of Benjamini and Schramm, which interpretes the equilibrium measure of a planar graph's boundary in terms of square tilings of cylinders.
\end{abstract}

Keywords Infinite trees $\cdot$ Nonlinear potential theory $\cdot$ Capacity $\cdot$ Equilibrium measures · Tilings

Mathematics Subject Classification $31 \mathrm{C} 15 \cdot 05 \mathrm{C} 63 \cdot 05 \mathrm{C} 05 \cdot 05 \mathrm{~B} 45 \cdot 52 \mathrm{C} 20$

\section{Introduction}

In Electrostatics, an amount of, say positive, electric charge free to move across a conductor $A$ in the Euclidean space will reach an equilibrium configuration $\mu$, which at the same time: (1) minimizes the energy $\mathcal{E}(\mu)$ carried by the generated electrostatic potential; (2) minimizes the maximum value of the potential; (3) makes the potential constant on the whole $A$, except possibly for a small exceptional set. For a given system of units, there is an amount $\|\mu\|$ of charge for which the potential on (most of) $A$ is unitary. The total charge $\|\mu\|$ is the capacity of the conductor and $\mu$ is the corresponding equilibrium measure of $A$. The mathematical theory of electrostatics, developed by Gauss, then put on firm mathematical foundations by Frostman, was later extended in many directions. See [6] for a survey of axiomatic linear theories which goes far beyond the scope of this article, and [1] for a rather general axiomatic nonlinear theory. The problem we consider here, in a special instance, is that of characterizing

The two authors are partially supported by the 2015 PRIN grant Real and Complex Manifolds: Geometry, Topology and Harmonic Analysis of the Italian Ministry of Education (MIUR)

Matteo Levi

matteo.levi@polito.it

Nicola Arcozzi

nicola.arcozzi@unibo.it

1 Dipartimento di Matematica, Università di Bologna, Piazza di Porta S. Donato, 5, 40127 Bologna, Italy

2 Dipartimento di Matematica, Politecnico di Torino, Corso Duca degli Abruzzi, 24, 10129 Torino, Italy 
equilibrium measures. Namely, given a positive measure $\mu$, our "measurable", is there a way to tell whether or not it is the equilibrium measure for some conductor $A$ ? The equilibrium measures are known to satisfy a number of properties, but to the best of our knowledge a complete answer is available only for the equilibrium measure of the boundary of a planar graphs. The case of finite, planar graphs is studied in [14], and a combinatorial interpretation of the equilibrium measure $\mu$ of the boundary of possibly infinite, planar graphs satisfying an extra technical hypothesis, is contained in a beautiful article by Benjamini and Schramm [4].

In this article, we characterize the equilibrium measures, within Nonlinear Potential Theory, for subsets of the boundary of an infinite tree. Benjamini and Schramm's theorem, in the case of trees, would apply to the equilibrium measures of a closed subset of the tree boundary. We find a condition which characterizes the equilibrium measures of subset of the trees, providing, in this special context, a converse to their result.

In order to state our main finding, we fix some minimal notation, to be better developed in the next section. A tree $T$ is a connected graph with no cycles. We denote by $E$ the set of its edges and by $V$ the set of its vertices. Given two vertices $x, y$, we write $x \sim y$ if they are connected by an edge. We consider infinite trees such that each vertex has a finite but arbitrary number of neighbors. We assume that there is a unique, distinguished edge $\omega$ one of whose endpoints, $o$, is not endpoint of any other edge. We say that $o$ is the root of $T$. Let \# denote the standard graph distance, which counts the number of edges along the shortest path connecting two vertices. A sequence of pairwise connected edges with no repetitions is called a geodesic, since it minimizes the graph distance between any couple of vertices lying along it. We also say that a vertex belongs to a geodesic if it is the endpoint of some edge belonging to the geodesic. A half infinite geodesic emanating from $o$ is said to be a ray. The boundary $\partial T$ of the tree can be classically identified with the set of rays. We set $\bar{V}=V \cup \partial T$. We partially order $\bar{V}$ by writing $\xi \geq y$ if and only if $y$ lies on the geodesic connecting $o$ and $\xi$ (or if $y$ belongs to the ray $\xi$ in the case $\xi \in \partial T$ ). Thus, edges are oriented and we can identify $E$ with the subset of couples $(x, y)$ in $V \times V$ such that $x \sim y$ and $y>x$. The boundary of the tree is a compact metric space with respect to the visual metric (see the section on preliminaries). We have then Borel signed measures on $\partial T$, which we call charges, while measures are intended to be nonnegative. As we will detail in the next section, using these ingredients and following the classical theory presented in [1], we can develop a Nonlinear Potential Theory on the tree. In particular, for any $p \in(1,+\infty)$ one can associate to a charge $\mu$ a nonlinear potential $V_{p} \mu: \bar{V} \rightarrow \mathbb{R}$, and a notion of $p$-capacity for subsets of the boundary is made available, see Sect. 1 for the definitions. By the general theory, we know that for each capacitable $A \subseteq \partial T$, there exists a unique positive measure $\mu^{A}$ with support in the closure of $A$ realizing its $p$-capacity $c_{p}(A)$, namely, such that $c_{p}(A)=\left\|\mu^{A}\right\|$. We call $\mu^{A}$ the $p$-equilibrium measure for $A$.

Equilibrium measures are strictly related to trace inequalities for discrete Hardy operators [2, Theorem 5]. The simplest interpretation, however, is in terms of elementary rescaling properties of trees, see Sect. 2. Our goal is characterizing which measures $\mu$ are equilibrium measures of some subset of the boundary. Such a characterization is encoded in the following integro-differential equation, which we will call, accordingly, the equilibrium equation:

$$
\nabla g[x, y]|\nabla g|^{p-2}[x, y](1-g(x))=\sum_{\substack{[z, w] \in E \\ z \geq x}}|\nabla g[z, w]|^{p}, \quad[x, y] \in E .
$$


In the above expression $p, p^{\prime} \in(1, \infty)$ are Hölder conjugate exponents, $E$ denotes the set of edges of the tree, $g$ is a function defined on the vertices, $\nabla g[x, y]:=g(y)-g(x)$ denotes its gradient on the edge $[x, y]$ and the right-hand side is a Sobolev energy. Our main result is the following.

Theorem 1 (i) Let $\mu$ be the $p$-equilibrium measure for a set $A \subseteq \partial T$. Then the function $g=V_{p} \mu$ solves $(1)$.

(ii) Conversely, let $g: V \rightarrow \mathbb{R}$ be a solution of (1) with $\|g\|_{\infty}<1$. Then, there exists an $\mathcal{F}_{\sigma}$ set $A \subseteq \partial T$ with equilibrium measure $\mu^{A}$ such that $g=V_{p}\left(\mu^{A}\right)$.

Observe that equation (1) is non linear even in Linear Potential Theory. This is not surprising, since linear combinations of equilibrium measures are only seldom equilibrium measures themselves. From the discussion in Sect. 3 it will be clear that solutions of (1) are automatically $p$-harmonic functions which are increasing along geodesics emanating from $o$. We will see how the equilibrium equation may be reformulated as an equation for measures (equation (4) in Sect. 2) or for edge functions (equation (5) in Sect. 3).

The equation can be interpreted in several ways. In the linear case $p=2$ it says that equilibrium measures on trees can be associated to particular tilings of rectangles by squares. This gives an independent proof of the tiling theorem of Benjamini and Schramm [4, Theorem 4.1], in the special case of a tree, and, more interestingly, it provides a converse result.

Here is the precise statement (see Sect. 5). We say that a square tiling of the rectangle $R=[0, t] \times[0,1]$ has combinatorics prescribed by a tree $T$ if there is a bijection $\alpha \mapsto Q_{\alpha}$ from the edge set of $T$ to the set of tiles such that $\alpha$ and $\beta$ have a common vertex and $\alpha<\beta$ if and only if the squares $Q_{\alpha}$ and $Q_{\beta}$ are neighbouring in the tiling and the upper side of $Q_{\beta}$ lies on the lower side of $Q_{\alpha}$.

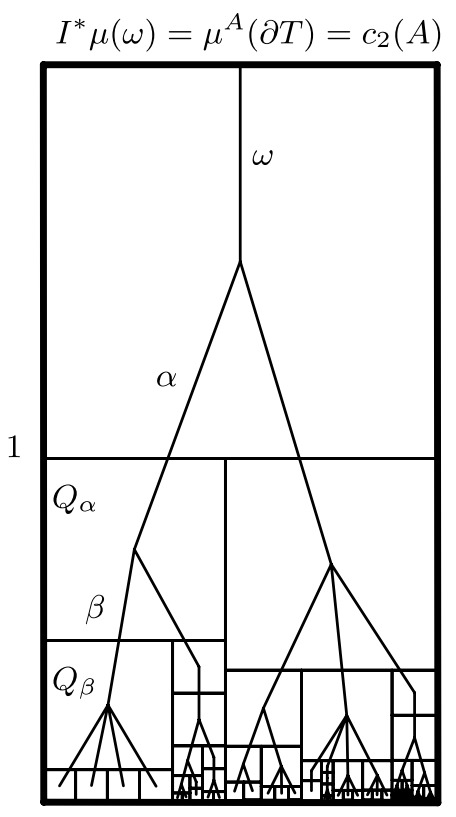


Theorem 2 (i) Let $T=(V, E)$ be a rooted tree and $\mu^{A}$ be the equilibrium measure (for $p=2)$ for a set $A \subseteq \partial T$.

Then, there exists a square tiling $\left\{Q_{\alpha}\right\}_{\alpha \in E}$ of the rectangle $R=\left[0, c_{2}(A)\right] \times[0,1]$, where the combinatorics of the tiling are prescribed by $T$ and the square $Q_{\alpha}$ has side of length $\ell(\alpha)=\mu^{A}\left(\partial T_{\alpha}\right)$.

(ii) Conversely, suppose a rectangle $R=[0, c] \times[0,1], c \leq 1$, is square-tiled by $\left\{Q_{\alpha}\right\}_{\alpha}$ with combinatorics given by a rooted tree $T$.

Then there exists an $F_{\sigma}$ subset $A$ of $\partial T$ such that the measure $\mu\left(\partial T_{\alpha}\right)=\ell(\alpha)$, is the equilibrium measure of $A$, and then $c_{2}(A)=c$.

A consequence of this theorem is that the different tilings having the same tree-combinatorics can be parametrized by a family of $F_{\sigma}$ subsets of $\partial T$.

Theorem 2 is very much related to results in [4, 12, 14]. Benjamini and Schramm proved that the equilibrium measure of a planar graph's boundary is associated to a tiling of a cylinder by squares, and on trees, analytically, this is the content of (1). The converse statement is not true: there are tilings of cylinders whose combinatorics are prescribed by a planar graph, in which the sizes of the tiles do not reflect the equilibrium measure of the graph's boundary; trees provide plenty of counterexamples. Theorem 2 provides, in the special case of trees, the correct bijection between tilings and discrete potential theoretic objects. It would be very interesting to have a generalization of this statement to the whole class of planar graphs.

Again in the linear case, Theorem 1 is related to some beautiful theorems of Kai-Lai Chung (see $[10,11]$ ), interpreting equilibrium measures in terms of last exit times for stochastic processes. The results of Chung, and of Benjamini and Schramm, have a probabilistic statement, or proof. As we work in the nonlinear case $1<p<\infty$, we do not expect probabilistic methods to apply here. Even in the linear case, however, it would be interesting to have a converse to Benjamini and Schramm's theorem on planar graphs, and this converse might have a probabilistic proof. See the monographs [13, 16] and [15] for thorough introductions to the stochastic processes which are here relevant.

The paper is organized as follows. Section 1 is devoted to present some preliminary results of Potential Analysis on trees. Section 2 contains the proof of (i) in Theorem 1, which follows quite easily from some rescaling properties we present. In Sect. 3 we provide some useful reinterpretation of equation (1) in terms of edge functions and of measures. The proof of part (ii) of our characterization is more subtle and is given in Sect. 4. In Sect. 5 we discuss some new results on square tilings of rectangles which follow from Theorem 1 with $p=2$. Finally, in Sect. 6 we show how capacities can be expressed by a recursive formula involving branched continued fractions, and we exploit this fact to give another reformulation of Theorem 1 . 


\section{Potential theory on the tree}

Let $T$ be a tree with root vertex $o$ and root edge $\omega$, as described in the Introduction. A vertex is said to be a leaf if it is the endpoint of one edge only. Being mainly interested in infinite trees, we will assume that $T$ has no leaves except $o$; this is a simplification but not a restriction. Given $\alpha \in E$, we write $b(\alpha)$ and $e(\alpha)$ for its beginning and ending vertex, respectively, i.e., they are connected by $\alpha$ and $b(\alpha)<e(\alpha)$. We denote by $|\alpha|$ the level of an edge $\alpha$, which is the number of edges preceding $\alpha$ in the geodesic to the root $\omega$. With this definition we have $|\omega|=0$. Levels of vertices are given by the rule $|b(\alpha)|=|\alpha|$. We define the sons of an edge $\alpha$ as the elements of the set $s(\alpha)=\{\beta \in E: \beta \sim \alpha,|\beta|=|\alpha|+1\}$. A subtree rooted at $\alpha$ is a rooted tree $S$ having root edge $\alpha$ and as edge set a subset of $\{\beta \in E: \beta \geq \alpha\}$, the set of edges of $T$ which are larger than $\alpha$ with respect to the order relation induced by $o$. We simply say that $S$ is a subtree of $T$ if it is a subtree rooted at $\omega$. We denote by $T_{\alpha}$ the full subtree rooted at $\alpha$, i.e., that whose edge set is exactly $\{\beta \in E: \beta \geq \alpha\}$. We call $T_{\alpha}$ the $\alpha$-tent. Observe that subtrees are obtained subtracting from $T$ a countable union of tents. The boundary $\partial T_{\alpha}$ of the $\alpha$-tent is exactly the set of rays passing through $\alpha$. We put on $\partial T$ the topology generated by the boundary of tents, namely the one having as a basis $\left\{\partial T_{\alpha}\right\}_{\alpha \in E}$.

It is clear that the counting metric \# does not extend properly to the boundary of an infinite tree. We endow $\bar{V}$ with a different metric: given two points $\xi, \eta \in \bar{V}$, we define their confluent to be the vertex $\xi \wedge \eta=\max \{x \in V: x \leq \xi, x \leq \eta\}$. Then, we define the visual metric

$$
d(\xi, \eta)=e^{-|\xi \wedge \eta|} .
$$

The reader familiar with Gromov's theory of hyperbolic spaces can observe that for $\xi, \eta \in V$, expression (2) coincides with the Gromov product on $(V$,\#), given by

$$
(x, y)_{o}=\frac{1}{2}(\#(x, o)+\#(y, o)-\#(x, y)), \quad x, y \in V .
$$

In fact, one can extend such a product to points in $\partial T$ by setting $(\xi, \eta)_{o}=\lim (x, y)_{o}$, where the limit is taken for $x \rightarrow \xi, y \rightarrow \eta$ along the rays labeled by $\xi$ and $\eta$. With this notation we have $d(\xi, \eta)=(\xi, \eta)_{o}$. It is not hard to see that (2) defines a distance on $\bar{V}$, which in fact is an ultrametric, and that $(\bar{V}, d)$ is a compact metric space (see, for example, [15, p. 121]). Moreover, the topology induced by this metric on $\partial T$ is the tent topology introduced before.

There is a one-to-one correspondence between compact sets in $\partial T$ and boundaries of subtrees, in the following sense.

Proposition 1 A set $K \subseteq \partial T$ is compact if and only if there exists a subtree $T_{K} \subseteq T$ such that $K=\partial T_{K}$.

Proof The fact that the boundary of a subtree is compact follows directly from the definition of subtree. Conversely, if $K$ is compact, consider the subtree $S \subseteq T$ having as edge set $E(S)=P(K):=\{\alpha \in E: \alpha<\xi$, for some $\xi \in \partial K\}$. Clearly $K \subseteq \partial S$. On the other hand, if $\xi \in \partial S$, by definition of boundary of a tree, $P(\{\xi\}) \subseteq E(S)$. Now, suppose by contraddiction that $\xi \notin K$. Then, by compactness, there exists and an edge $\alpha \in E$ such that $\partial T_{\alpha} \bigcap K=\emptyset$ and $\xi \in \partial T_{\alpha}$. Hence, $\beta \notin P(K)$ for $\beta \in P(\{\xi\}) \subseteq E(S)$ with $|\beta| \geq|\alpha|$, leading to a contraddiction. 
We present now a Nonlinear Potential Theory on the tree $T$ which falls within the axiomatics developed in \$2.3-2.5 of the treatise of Adams-Hedberg [1]. We consider the compact metric space $(\bar{V}, d)$ and we make $E$ into a measure space by endowing it with the counting measure. We introduce the kernel $k: \bar{V} \times E \rightarrow \mathbb{R}$, given by the indicator function $k(\xi, \alpha)=\mathbb{q}_{\{\alpha<\xi\}}(\xi, \alpha)$. Observe that $k(\cdot, \alpha)$ is continuous on $\partial T$, since $\partial T_{\alpha}$ is open.

Given a function $f: E \rightarrow \mathbb{R}$ and $p, p^{\prime} \in(1,+\infty)$ such that $1 / p+1 / p^{\prime}=1$, we define the $p$-potential of $f, I_{p} f: \bar{V} \rightarrow \mathbb{R} \cup\{ \pm \infty\}$, by

$$
I_{p} f(\xi)=\sum_{\alpha \in E} k(\xi, \alpha) f(\alpha)|f(\alpha)|^{p^{\prime}-2}=\sum_{E \ni \alpha<\xi} f(\alpha)|f(\alpha)|^{p^{\prime}-2} .
$$

We fix the convention $I_{p} f(o)=0$ and we ease the notation by simply writing If in place of $I_{2} f$.

The co-potential of a charge $\mu \in \mathcal{M}(\partial T)$ is defined as the edge function

$$
I^{*} \mu(\alpha)=\int_{\bar{V}} k(\xi, \alpha) d \mu(\xi)=\mu\left(\partial T_{\alpha}\right), \quad \alpha \in E .
$$

Observe that $\langle\text { If }, \mu\rangle_{L^{2}(\partial T)}=\left\langle f, I^{*} \mu\right\rangle_{\ell^{2}(E)}$.

To the charge $\mu$ one can then associate the nonlinear potential $V_{p} \mu(\xi)=I_{p} I^{*} \mu(x)$, $\xi \in \bar{V}$. It is easily seen that for $p=2$ one recovers the logarithmic potential of $\mu$ on the metric space $(\bar{V}, d)$,

$$
V_{2} \mu(\xi)=\int_{\partial T} \log \frac{1}{d(\xi, v)} d \mu(v), \quad \xi \in \bar{V}
$$

The $p$-energy of the charge $\mu$ is given by

$$
\mathcal{E}_{p}(\mu)=\int_{\partial T} V_{p} \mu(\xi) d \mu(\xi)=\left\|I^{*} \mu\right\|_{p^{\prime}}^{p^{\prime}} .
$$

Setting $\Omega_{A}=\left\{f \in \ell^{p}(E):\right.$ If $\geq 1$ on $\left.A\right\}$, we define the $p$-capacity of a set $A \subseteq \partial T$ as

$$
c_{p}(A)=\inf _{f \in \Omega_{A}}\|f\|_{p}^{p} .
$$

A property holds $c_{p}$-a.e. on $A$ if it holds everywhere on $A$ but for a subset of zero p-capacity. A function $f$ is admissible for $A \subseteq \partial T$ if $f \in \overline{\Omega_{A}}$. It can be proved that ${\overline{\Omega_{A}}}=\left\{f_{p} \in \ell_{+}^{p}:\right.$ If $\geq 1 c_{p}-$ a.e. on $\left.A\right\}$, see [1], and that there exists a unique function $f^{A} \in \overline{\Omega_{A}}$, called $p$-equilibrium function for $A$, such that

$$
c_{p}(A)=\min _{f \in{\overline{\Omega_{A}}}^{p}}\|f\|_{p}^{p}=\left\|f^{A}\right\|_{p}^{p} .
$$

Moreover, for such a function it holds $I f^{A}=1 c_{p}$-a.e. on $A$.

We call a point $\xi \in A$ irregular for $A$ if $I f^{A}(\xi) \neq 1$. Clearly the set of irregular points for any set $A$ has zero $p$-capacity.

As a set function, $p$-capacity is monotone, countably subadditive and regular from inside and outside, i.e.,

(in) $\quad c_{p}\left(\bigcup A_{n}\right)=\lim _{n} c_{p}\left(A_{n}\right)$ for any increasing sequence $\left(A_{n}\right)$ of arbitrary subsets of $\partial T$ (out) $c_{p}\left(\bigcap_{n}^{n}\left(K_{n}\right)\right)=\lim _{n} c_{p}\left(K_{n}\right)$, for any decreasing sequence $\left(K_{n}\right)$ of compact subsets of $\partial T$ 
A set $A \subseteq \partial T$ is capacitable if $c_{p}(A)=\sup \left\{c_{p}(K): K \subseteq A, K\right.$ compact $\}=\inf \left\{c_{p}(G): G \supseteq A, G\right.$ open $\}$. From (in) and (out) follows that all Suslin sets, and in particular all Borel sets, are capacitable, see [9].

Observe that without losing generality, in the definition of $p$-capacity the infimum can be taken over functions supported on the predecessor set of $\bar{A}$, $P(\bar{A}):=\{\alpha \in E: \alpha<\xi$, for some $\xi \in \bar{A}\}$. Namely, the capacity of a compact set $K$ only depends on the combinatorics of $P(K)$ and not on the rest of the tree, and if $T_{K} \subseteq T$ is the subtree having $K$ as a boundary (see Proposition 1), we have $c_{p}(K)=c_{p}\left(\partial T_{K}\right)$, where the right handside is intended as the capacity when $T_{K}$ is taken as the ambient space.

It is possible to give a dual definition of capacity in terms of measures on $\partial T$ rather than of functions on $E$. The proof of the following theorem is a straightforward adaptation of an equivalent result [ 1 , Theorem 2.5 .6$]$ for capacities in $\mathbb{R}^{n}$ arising by regular enough kernels.

Theorem A Suppose that $A \subseteq \partial T$ is a Suslin set. Then

$$
c_{p}(A)=\sup \left\{\mu(A)^{p}: \mu \geq 0, \quad \operatorname{supp}(\mu) \subseteq A, \mathcal{E}_{p}(\mu) \leq 1\right\} .
$$

Moreover, there exists a unique positive Borel measure $\mu^{A}$ supported in $\bar{A}$, called the $p-$ equilibrium measure of $A$, such that

$$
\mu^{A}(\bar{A})=c_{p}(A)=\mathcal{E}_{p}\left(\mu^{A}\right),
$$

and $I^{*} \mu^{A}(\alpha)^{p^{\prime}-1}=f^{A}(\alpha), \alpha \in E$.

With this definition of capacity, it is clear that if a property $(P)$ holds $c_{p}$-a.e. on $A \subseteq \partial T$, then it holds $\mu$-a.e. on $A$ for every measure $\mu$ with $\mathcal{E}_{p}(\mu)<\infty$. To see this, let $B:=\{\xi \in A: \neg(P)\}$, so that $c_{p}(B)=0$. The measure $v:=\frac{\left.\mu\right|_{B}}{\mathcal{E}_{p}(\mu)^{1 / p^{\prime}}}$ satisfies $\mathcal{E}_{p}(\nu) \leq 1$. Then, $v(B) \leq c_{p}(B)=0$, from which it follows $\mu(B)=0$.

We conclude the section by showing that in $\partial T$ there exist compact subsets with arbitrary $p$-capacity.

Proposition 2 Let $T^{n}$ be a homogeneus tree of degree $n$. For each real number $t \in\left[0, c_{p}\left(\partial T^{n}\right)\right]$ there exists a compact subset $K_{t}$ of the boundary $\partial T^{n}$ such that $c_{p}\left(K_{t}\right)=t$.

Proof Each edge $\alpha$ of $T^{n}$, except the root, can be given an index $i(\alpha) \in\{0, \ldots, n-1\}$ which distinguishes it from the other $n-1$ edges $\beta$ such that $b(\beta)=b(\alpha)$. We can define a map $\Lambda: \partial T^{n} \rightarrow[0,1]$ associating to each point $\xi=\left\{\alpha_{j}\right\}_{j=1}^{\infty} \in \partial T^{n}$ the number having expansion in base $n$ given by

$$
\Lambda(\xi)=\sum_{j=1}^{\infty} i\left(\alpha_{j}\right) n^{-j} .
$$

The map $\Lambda$ is clearly onto but it fails to be injective because of the multiple representations of the rational numbers. Still, $\Lambda^{-1}(t)$ has at most two points. Moreover, $\Lambda$ is continuous, since 


$$
|\Lambda(\xi)-\Lambda(\eta)| \leq(n-1) \sum_{j=|\xi \wedge \eta|+1}^{\infty} n^{-j} \approx n^{-|\xi \wedge \eta|} \longrightarrow 0, \quad \text { as } \rho(\xi, \eta) \rightarrow 0 .
$$

Now, consider the function $\varphi:[0,1] \longrightarrow \mathbb{R}$ given by $\varphi(t)=c_{p}\left(\Lambda^{-1}[0, t]\right)$. This is an increasing map, and we know that $\varphi(0)=0$ and $\varphi(1)=c_{p}\left(\partial T^{n}\right)$. By the subadditivity of $c_{p}$, the continuity of $\Lambda$ and the regularity of capacity, we have

$$
\varphi(t+\varepsilon)-\varphi(t) \leq c_{p}\left(\Lambda^{-1}[t, t+\varepsilon]\right) \longrightarrow c_{p}\left(\Lambda^{-1}\{t\}\right) .
$$

The right handside equals zero, since the preimage of a single point under $\Lambda$ is finite. By similar reasoning we estimate $\varphi(t)-\varphi(t-\epsilon)$. It follows that $\varphi$ is continuous and $\varphi([0,1])=\left[0, c_{p}\left(\partial T^{n}\right)\right]$. The result is obtained picking $K_{t}=\Lambda^{-1}\left[0, \varphi^{-1}(t)\right]$.

\section{Rescaling of capacities}

In this section we show that equilibrium measures rescale under changes of the root in a tree, in a sense that will be more clear soon. This is a point where trees behave much more simply than general planar graphs, and constitutes the key property to prove $(i)$ in Theorem 1. We introduce a subscript notation to indicate which is the root of the tree we are referring to. For example, given the rooted tree $T$ and some edge $\alpha \in E$, we write $I_{p, \alpha}$ for the $p$-potential operator acting on functions defined on the edges of the $\alpha$-tent $T_{\alpha}$, $I_{p, \alpha} f=I_{p}\left(\chi_{T_{\alpha}} f\right)$. Accordingly, $V_{p, \alpha} \mu=I_{p, \alpha} I^{*} \mu, \mathcal{E}_{p, \alpha}(\mu)=\sum_{\beta \geq \alpha}\left|I^{*} \mu(\beta)\right|^{p^{\prime}}$ and $c_{p, \alpha}$ denotes the capacity when we consider $T_{\alpha}$ as ambient space. In the same fashion, if $A$ is a subset of $\partial T, A_{\alpha}=A \cap \partial T_{\alpha}$.

A question arises: if $A$ is a subset of $\partial T$ and $\mu$ its $p$-equilibrium measure, which is the $p$-equilibrium measure $\mu_{\alpha}$ for $A_{\alpha}$ in the tent $T_{\alpha}$ ? It is natural to expect that it is a rescaling of the measure $\mu$, i.e., $\mu_{\alpha}=\left.k_{\alpha} \mu\right|_{A_{\alpha}}$ for some positive constant $k_{\alpha}$. In such a case, for $c_{p}$-a.e. $\xi$ in $A_{\alpha}$, we would have

$$
1=V_{p, \alpha} \mu_{\alpha}(\xi)=k_{\alpha}^{p^{\prime}-1} V_{p, \alpha} \mu(\xi)=k_{\alpha}^{p^{\prime}-1}\left(V_{p} \mu(\xi)-V_{p} \mu(b(\alpha))\right)=k_{\alpha}^{p^{\prime}-1}\left(1-V_{p} \mu(b(\alpha))\right) .
$$

It follows that the only possible candidate rescaling constant is

$$
k_{\alpha}=\left(1-V_{p} \mu(b(\alpha))\right)^{1-p}
$$

We now prove that our Ansatz is correct. This was already observed in [3].

Proposition 3 Let $\mu$ be the $p$-equilibrium measure for a set $A \subseteq \partial T$. Then,

$$
\mu_{\alpha}:=\left.k_{\alpha} \mu\right|_{A_{\alpha}}=\frac{\left.\mu\right|_{\partial T_{\alpha}}}{\left(1-V_{p} \mu(b(\alpha))\right)^{p-1}},
$$

is the p-equilibrium measure for $A_{\alpha} \subseteq \partial T_{\alpha}$. 
Proof Let $\varphi_{\alpha}$ be the (unique) $p$-equilibrium function of $A_{\alpha}$ in $T_{\alpha}$. We want to prove that $\varphi_{\alpha}=I^{*} \mu_{\alpha}^{p^{\prime}}-1^{\alpha}$. Define the edge function,

$$
f(\beta)= \begin{cases}\left(1-V_{p} \mu(b(\alpha))\right) \varphi_{\alpha}(\beta) & \text { if } \beta \geq \alpha \\ I^{*} \mu(\beta)^{p^{\prime}-1} & \text { otherwise } .\end{cases}
$$

Then $f$ is admissible for $A \subseteq \partial T$, since for $c_{p}$-a.e. $\xi \in A \backslash A_{\alpha}, I f(\xi)=V_{p} \mu(\xi)=1$, while for $c_{p}$-a.e. $\xi \in A_{\alpha}$ it holds

$$
I f(\xi)=I_{\alpha} f(\xi)+I f(b(\alpha))=\left(1-V_{p} \mu(b(\alpha))\right) I_{\alpha} \varphi_{\alpha}(\xi)+V_{p} \mu(b(\alpha))=1 .
$$

Clearly $I^{*} \mu^{p^{\prime}-1}$ is an admissible function for $A_{\alpha} \subseteq \partial T_{\alpha}$, since $I_{\alpha} I^{*} \mu_{p^{\prime}-1}^{p^{\prime}} \leq I I^{*} \mu^{p^{\prime}-1}=1, c \cdot p-$ a.e. on $A$. Hence, it must be $\left\|\varphi_{\alpha}\right\|_{\ell p^{p}\left(T_{\alpha}\right)}^{p} \leq\left\|I^{*} \mu^{p^{\prime}-1}\right\|_{\ell^{p}\left(T_{\alpha}\right)}^{p}=\left\|I^{*} \mu\right\|_{\ell^{p^{\prime}}\left(T_{\alpha}\right)}^{p^{\prime}}$. It follows

$$
\begin{aligned}
\|f\|_{p}^{p} & =\left(1-V_{p} \mu(b(\alpha))\right)^{p}\left\|\varphi_{\alpha}\right\|_{\ell p\left(T_{\alpha}\right)}^{p}+\left\|I^{*} \mu^{p^{\prime}-1}\right\|_{\ell p\left(T \backslash T_{\alpha}\right)}^{p} \\
& \leq\left\|I^{*} \mu\right\|_{\ell p^{\prime}\left(T_{\alpha}\right)}^{p^{\prime}}+\left\|I^{*} \mu\right\|_{\ell p^{\prime}\left(T \backslash T_{\alpha}\right)}^{p^{\prime}}=\mathcal{E}_{p}(\mu)=c_{p}(A) .
\end{aligned}
$$

Then, $f$ must be the equilibrium function for $A$, and by the uniqueness of the equilibrium function $f=I^{*} \mu^{p^{\prime}-1}$. This implies $\varphi_{\alpha}=I^{*} \mu_{\alpha}^{p^{\prime}-1}$.

As an immediate consequence we have the following.

Corollary 1 Let $A \subseteq \partial T$ be a set of positive capacity and $\alpha \in E, \alpha$ not the root edge of $T$, such that $A \subseteq \partial T_{\alpha}$. Then $c_{p, \alpha}(A)>c_{p}(A)$.

Proof Since $k_{\alpha}>1$, we have $c_{p, \alpha}(A)=\mu_{\alpha}(A)=k_{\alpha} \mu(A)>\mu(A)=c_{p}(A)$.

Observe in passing that the above corollary is supported by visual intuition, since we expect the capacity of a set to be larger if we look at the set from a closer point of view.

We can now give a necessary condition for a measure to be of equilibrium, thus proving $(i)$ in Theorem 1.

Proof (Proof of (i) in Theorem 1) We claim that for every $\alpha \in E, \mu$ solves the following equation:

$$
I^{*} \mu(\alpha)\left(1-V_{p} \mu(b(\alpha))\right)=\mathcal{E}_{p, \alpha}(\mu)
$$

Suppose the claim holds and let $g=V_{p} \mu$. It is easily seen that $\nabla g[x, y]=I^{*} \mu[x, y]^{p^{\prime}-1}$ for every edge $[x, y] \in E$. It follows that $g$ solves the equilibrium equation (1).

To prove the claim, let $\alpha \in E$. If $A_{\alpha}=\emptyset$, then by the topology of the tree follows that also $\bar{A} \cap \partial T_{\alpha}=\emptyset$. Since $\operatorname{supp}(\mu) \subseteq \bar{A}, I^{*} \mu(\alpha)=0$, and hence $I^{*} \mu(\beta)=0$ for all $\beta \geq \alpha$, and (4) trivially holds. Otherwise, on one hand we have

$$
c_{p, \alpha}\left(A_{\alpha}\right)=\mathcal{E}_{p, \alpha}\left(\mu_{\alpha}\right)=\frac{\mathcal{E}_{p, \alpha}(\mu)}{\left(1-V_{p} \mu(b(\alpha))\right)^{p}},
$$


and on the other,

$$
c_{p, \alpha}\left(A_{\alpha}\right)=\mu_{\alpha}\left(A_{\alpha}\right)=k_{\alpha} \mu\left(A_{\alpha}\right)=\frac{I^{*} \mu(\alpha)}{\left(1-V_{p} \mu(b(\alpha))\right)^{p-1}} .
$$

Matching the two expressions we get the claim.

\section{Flows on edges and $p$-harmonic functions}

Observe that equation (4) can be regarded as a special instance of an equation for edge functions:

$$
f(\alpha)\left(1-I_{p} f(b(\alpha))\right)=\sum_{\beta \geq \alpha}|f(\beta)|^{p^{\prime}}, \quad f: E \rightarrow \mathbb{R} .
$$

In the particular case when the function $f$ is the co-potential of a charge $\mu$ on the $\partial T$, the above equation reduces to (4). It is clear that a necessary condition for an edge function $f$ to be the co-potential of a charge is to satisfy the following additivity condition

$$
f(\alpha)=\sum_{\beta \in s(\alpha)} f(\beta), \quad \text { for all } \alpha \in E .
$$

A function $f: E \rightarrow \mathbb{R}$ fulfilling (6) is said to be a flow on $T$.

In [8, Proposition 2.2] a full characterization for edge functions which are co-potentials of charges is given. For nonnegative functions, however, such a characterization takes a very simple form, of immediate verification: an edge function $f$ is a nonnegative flow if and only if $f=I^{*} \mu$ for some nonnegative measure $\mu$ on $\partial T$.

It turns out that solutions of (5) with bounded $p$-potential are always flows, and hence they have a measure representation.

Proposition 4 Let $f: E \rightarrow \mathbb{R}$ be a solution of (5) such that $I_{p} f<1$ on $V$. Then $f=I^{*} \mu$ for some nonnegative measure $\mu$ on $\partial T$.

Proof First of all observe that for a function $f$ solving (5), the condition $I_{p} f<1$ automatically implies that $f \geq 0$ on $E$. We want to show that $f$ satisfies (6) for every $\alpha \in E$. If $f(\alpha)=0$ for some edge $\alpha$, then the right handside of equation (5) says that $f(\beta)=0$ on all edges $\beta \geq \alpha$ and then clearly (6) holds in $\alpha$. Now, consider edges $\alpha$ such that $f(\alpha) \neq 0$. We have

$$
\begin{aligned}
\sum_{\beta \geq \alpha} f(\beta)^{p^{\prime}}-f(\alpha)^{p^{\prime}} & =\sum_{\beta \in s(\alpha)} \sum_{\gamma \geq \beta} f(\beta)^{p^{\prime}}=\sum_{\beta \in s(\alpha)} f(\beta)\left(1-I_{p} f(b(\beta))\right) \\
& =\left(1-I_{p} f(e(\alpha))\right) \sum_{\beta \in s(\alpha)} f(\beta) \\
& =\left(1-I_{p} f(e(\alpha))\right)\left(-f(\alpha)+\sum_{\beta \in s(\alpha)} f(\beta)\right)+\sum_{\beta \geq \alpha} f(\beta)^{p^{\prime}}-f(\alpha)^{p^{\prime}} .
\end{aligned}
$$

Since $1-I_{p} f(e(\alpha))>0$, for every $\alpha$, then $f$ is a flow, and the result follows. 
A similar calculation as in the proof of Proposition 4 can be used to show that, if $f$ is a (not necessarily positive) flow and (4) holds for $|\alpha|$ large, then it holds everywhere. We give here the explicit details.

Proposition 5 Let $\alpha \in E$ and suppose that $f$ is a flow solving (4) for $\beta \in s(\alpha)$. Then $f$ solves (4) also in $\alpha$.

Proof Following the line of (7) and using the flow property of $f$ we easily obtain

$$
\begin{aligned}
\sum_{\beta \geq \alpha}|f(\beta)|^{p^{\prime}} & =|f(\alpha)|^{p^{\prime}}+\left(1-I_{p} f(e(\alpha))\right) \sum_{\beta \in s(\alpha)} f(\beta) \\
& =f(\alpha)\left(f(\alpha)|f(\alpha)|^{p^{\prime}-2}+1-I_{p} f(e(\alpha))\right)=f(\alpha)\left(1-I_{p} f(b(\alpha))\right) .
\end{aligned}
$$

We end the section by pointing out that the flow condition (6) for edge functions has a neat counterpart for vertex functions. In fact, it is easy to observe [8, Proposition 2.3] that $f$ is a flow if and only if $I_{p} f$ is $p$-harmonic on $V \backslash\{o\}$. In this context, the $p$-Laplacian of a function $g: V \rightarrow \mathbb{R}$ at a vertex $x$ is given by

$$
\Delta_{p} g(x):=\sum_{y \sim x}(g(y)-g(x))|g(y)-g(x)|^{p-2},
$$

and $g$ is $p$-harmonic if $\Delta_{p} g \equiv 0$ on $V \backslash\{o\}$. From Proposition 4 then immediately follows

Corollary 2 Let $g: V \rightarrow \mathbb{R}$ be a solution of (1) with $\|g\|_{\infty}<1$. Then $g$ is $p$-harmonic.

\section{Proof of Theorem 1}

In this section, we prove (ii) in Theorem 1, namely that for a measure with bounded nonlinear potential it is sufficient to solve equation (1) to be an equilibrium measure.

Proof (Proof of (ii) in Theorem 1) Let $g: V \rightarrow \mathbb{R}$, with $\|g\|_{\infty}<1$, be a solution of (1). Without loss of generality, normalize assuming $g(o)=0$ (the function $\tilde{g}=(g-g(o)) /(1-g(o))$ solves (1), is bounded by 1 and vanishes at $o$ ). Set $f(\alpha)=\nabla g(\alpha)^{p-1}, \alpha \in E$. Then $f$ solves (5) and $I_{p} f=g$ is bounded by 1 . It follows by Proposition 4 that there exists a nonnegative Borel measure $\mu$ on $\partial T$ such that $f=I^{*} \mu$ and, consequently, $g=V_{p} \mu$.

Observe that $\mu$ has finite $p$-energy: since it solves (4) at every edge $\alpha$, by evaluating at $\alpha=\omega$ we get $\mathcal{E}_{p}(\mu)=\mu(\partial T)=V_{p}(\mu)(e(\omega))^{p-1}<1$. Moreover, $V_{p} \mu(\xi)=\lim _{x \rightarrow \xi} V_{p} \mu(x) \leq 1$. We show that indeed $V_{p} \mu=1, \mu$-a.e. on $\partial T$.

Let $S_{N}=\{\alpha \in E:|\alpha|=N\}$. To each $N \in \mathbb{N}$ we associate a piecewise-constant function $\Phi_{N}$ on the boundary, $\Phi_{N}(\xi)=\left(1-V_{p} \mu(b(\alpha))\right)$ for $\xi \in \partial T_{\alpha}, \alpha \in S_{N}$. Then we have 


$$
\begin{aligned}
0 & \leq \int_{\partial T} \Phi_{N}(\xi) d \mu(\xi)=\sum_{\alpha \in S_{N}} \int_{\partial T_{\alpha}} \Phi_{N}(\xi) d \mu(\xi)=\sum_{\alpha \in S_{N}}\left(1-V_{p} \mu(b(\alpha))\right) I^{*} \mu(\alpha) \\
& =\sum_{\alpha \in S_{N}} \mathcal{E}_{p, \alpha}(\mu)=\sum_{|\beta| \geq N} I^{*} \mu(\beta)^{p^{\prime}}=\mathcal{E}_{p}(\mu)-\sum_{|\beta|<N} I^{*} \mu(\beta)^{p^{\prime}} .
\end{aligned}
$$

Since $\mathcal{E}_{p}(\mu)<\infty$, it follows that

$$
\int_{\partial T} \Phi_{N}(\xi) d \mu(\xi) \longrightarrow 0, \text { as } N \rightarrow+\infty
$$

Also, $\Phi_{N}(\xi) \searrow \Phi(\xi):=1-V_{p} \mu(\xi) \geq 0$ as $N \rightarrow+\infty$. Hence, by monotone convergence theorem, we obtain

$$
\int_{\partial T} \Phi(\xi) d \mu(\xi)=\int_{\partial T}\left(1-V_{p} \mu(\xi)\right) d \mu(\xi)=0,
$$

which gives $V_{p} \mu(\xi)=1, \mu-$ a.e. on $\partial T$.

Consider now the irregular points for $\mu$, i.e., with the $\mu$-measure zero set

$$
\mathcal{I}(\mu)=\left\{\xi \in \operatorname{supp}(\mu): V_{p} \mu(\xi)<1\right\} .
$$

Let $B_{n}=\left\{\xi \in \operatorname{supp}(\mu): V_{p} \mu(\xi) \leq 1-1 / 2^{n}\right\}$. Clearly $B_{n} \subseteq B_{n+1}$ and $\mathcal{I}(\mu)=\bigcup_{n} B_{n}$. Fix $\varepsilon>0$, and choose a collection of edges $\left(\alpha_{j}^{n}\right)_{n \in \mathbb{N}, j \in \mathcal{J}_{n}}$, such that $\left\{\partial T_{j, n}\right\}_{j \in \mathcal{J}_{n}}$ is an open cover of $B_{n}$, where $T_{j, n}$ is the $\alpha_{j}^{n}$-tent. Without loss of generally, we can assume that $\left(\alpha_{j}^{n}\right)_{n \in \mathbb{N}, j \in \mathcal{J}_{n}}$ satisfies the following:

(i) $B_{n} \subseteq \bigcup_{j \in \mathcal{J}_{n}} \partial T_{j, n}$

(ii) $T_{j, n} \bigcap T_{i, l}=\emptyset$, for $(j, n) \neq(i, l)$

(iii) $\left|\mathcal{J}_{n}\right|=m_{n} \in \mathbb{N}$

(iv) $\sum_{j \in \mathcal{J}_{n}} I^{*} \mu\left(\alpha_{j}^{n}\right)<\varepsilon / 2^{n}$.

In fact, if the intersection in (ii) was not empty, one of the two would be contained in the other and can be replaced by it in the covering family. Moreover, all the sublevel sets $B_{n}$ are compact, since $V_{p} \mu$ is continuous. Hence, for each $n$, we can extract a finite subcover so that $B_{n} \subseteq \bigcup_{j \in \mathcal{J}_{n}} \partial T_{j, n}$ with $\left|\mathcal{J}_{n}\right|=m_{n} \in \mathbb{N}$, which is the finiteness condition on the index set in (iii). Finally, condition (iv) comes from the outer regularity of the measure $\mu$ : since $0=\mu\left(B_{n}\right)=\inf \left\{\mu\left(\partial T_{\alpha}\right): \alpha \in E, \partial T_{\alpha} \supseteq B_{n}\right\}$, there exist sequences $\left(\alpha_{j}^{n}\right)_{j}$ such that $\mu\left(\partial T_{j, n}\right) \rightarrow 0$ and we can assume to properly extract each subcover from one of those.

Write $\partial T=F_{\varepsilon} \bigcup G_{\varepsilon}$, where $G_{\varepsilon}:=\bigcup_{j, n} \partial T_{j, n}$ and $F_{\varepsilon}=\partial T \backslash G_{\varepsilon}$. Observe that,

$$
\mu(\partial T) \geq \mu\left(F_{\varepsilon}\right)=\mu(\partial T)-\mu\left(G_{\varepsilon}\right)=\mu(\partial T)-\sum_{j, n} I^{*} \mu\left(\alpha_{j}^{n}\right) \geq \mu(\partial T)-\sum_{n} \varepsilon / 2^{n}=\mu(\partial T)-\varepsilon .
$$

Hence we have,

$$
\mu(\partial T)=\lim _{\varepsilon \rightarrow 0} \mu\left(F_{\varepsilon}\right) .
$$

Now, $V_{p} \mu \equiv 1$ on $A_{\varepsilon}:=\operatorname{supp}(\mu) \cap F_{\varepsilon}$, so that $V_{p} \mu$ is a $p$-admissible function for $A_{\varepsilon}$. Then, by definition of capacity we have 


$$
c_{p}\left(A_{\varepsilon}\right) \leq \mathcal{E}_{p}\left(\left.\mu\right|_{F_{\varepsilon}}\right) \leq \mathcal{E}_{p}(\mu)=\mu(\partial T) .
$$

On the other hand, the measure $v^{\varepsilon}:=\frac{\left.\mu\right|_{F_{\varepsilon}}}{\mathcal{E}_{p}\left(\left.\mu\right|_{F_{\varepsilon}}\right)^{1 / p^{\prime}}}$ is admissible for $A_{\varepsilon}$, since $\mathcal{E}_{p}\left(\nu^{\varepsilon}\right)=1$. By the dual definition of capacity it follows that

$$
c_{p}\left(A_{\varepsilon}\right) \geq \nu^{\varepsilon}\left(A_{\varepsilon}\right)^{p} \geq\left(\frac{\mu\left(F_{\varepsilon}\right)}{\mu(\partial T)^{1 / p^{\prime}}}\right)^{p} .
$$

We are now ready to build up the candidate $F_{\sigma}$ set. Let $\{\varepsilon(k)\}_{k \in \mathbb{N}}$ be a sequence of positive numbers such that $\varepsilon(k) \searrow 0$ as $k \rightarrow+\infty$. Define $A:=\bigcup_{k} A_{\varepsilon(k)}$, which is clearly an $F_{\sigma}$ set. Observe that we can assume that the covers related to each choice of $\varepsilon$ are taken so that $G_{\varepsilon(k+1)} \subseteq G_{\varepsilon(k)}$. Therefore, $A_{\varepsilon(k)} \nearrow$ as $k \rightarrow+\infty$. It follows that

$$
\mu(A)=\lim _{k \rightarrow \infty} \mu\left(A_{\varepsilon(k)}\right)=\mu(\partial T)-\lim _{k \rightarrow \infty} \mu\left(G_{\varepsilon(k)}\right) \geq \mu(\partial T)-\lim _{k \rightarrow \infty} \sum_{n} \frac{\varepsilon(k)}{2^{n}}=\mu(\partial T),
$$

while the reverse inequality is trivially true. Using this together with (8) and (9) and the regularity of the $p$-capacity, we obtain

$$
c_{p}(A)=\lim _{k \rightarrow+\infty} c_{p}\left(A_{\varepsilon(k)}\right)=\mu(\partial T)=\mu(A) .
$$

It is clear from the proof that the situation is much easier for measures with no irregular points.

Corollary 3 Suppose that $\mu \in \mathcal{M}^{+}(\partial T)$ solves (4) and $V_{p} \mu \equiv 1$ on $\operatorname{supp}(\mu)$. Then, $\mu$ is the p-equilibrium measure of $\operatorname{supp}(\mu)$.

\section{Infinite square tilings}

In [7] Brooks, Smith, Stone and Tutte considered the problem of tiling a rectangle with a finite number of squares and proved that to any finite connected planar graph $G$ can be associated such a tiling. The same graph can produce different tilings. Chosen any two vertices in $G$, they show how the associated tiling can be built in such a way to reflect this choice. In [4] Benjamini and Schramm extended this result to the infinite case, showing that infinite graphs can produce infinite tilings. Theorem 1 can be reformulated, for $p=2$, in terms of square tilings of a rectangle: part $(i)$ of the theorem is essentially equivalent to the infinite tiling theorem by Benjamini and Schramm, in the special case when $G$ is a rooted tree $T$, hence providing a new and different proof of it. More interestingly, part (ii) provides a converse result, in a sense that will be more clear once we introduce the proper terminology.

Given a rectangle $R$, i.e, a closed planar region whose boundary is a rectangle, we say that a family of squares $Q=\left\{Q_{j}\right\}_{j}$ is a square tiling of $R$ if int $\left(Q_{i}\right) \bigcap \operatorname{int}\left(Q_{j}\right)=\emptyset$, for $i \neq j$, and $R=\bigcup_{j} Q_{j}$. By rotation invariance of the problem we always think rectangles and squares to have sides parallel to the coordinates axes of $\mathbb{R}^{2}$, and we talk about upper (lower) 
and left (right) sides, as well as horizontal and vertical sides, in the obvious way. We write $B(j)$ and $E(j)$ for the upper and lower side of $Q_{j}$, respectively.

We say that the combinatorics of a family $Q$ of squares in the plane are prescribed by a tree $T$ if the followings are true.

(1) The squares in the family are indexed by the edges of the tree, $Q=\left\{Q_{\alpha}: \alpha \in E\right\}$.

(2) $B(\alpha) \subseteq E(\beta)$ whenever $b(\alpha)=e(\beta)$.

Theorem 1 can be reformulated, for $p=2$, in terms of square tilings, leading to Theorem 2. We detail out the proof here.

Proof (Proof of Theorem 2) (i) Given a tree $T$ with root edge $\omega$ and a set $A \subseteq \partial T$, let $\left\{Q_{\alpha}\right\}_{\alpha \in E}$ be a family of squares such that $Q_{\alpha}$ has side of length $\ell(\alpha)=\mu\left(\partial T_{\alpha}\right)$, being $\mu=\mu^{A}$ the equilibrium measure of $A$. By the additivity of $\mu$ we can place the squares on the plane in such a way that,

$$
E(\beta)=\bigcup_{\beta \in s(\alpha)} B(\alpha) .
$$

With this choice, the combinatorics is prescribed by $T$. Moreover, it is clear that the interiors of the squares in the family are pairwise disjoint and that $\bigcup_{\alpha} Q_{\alpha}$ is both vertically and horizontally convex (its intersection with any vertical and horizontal line is either empty, or a point, or a line segment). Now, let $R$ be the rectangle having vertical sides of length 1 and upper side coinciding with the upper side of $Q(\omega)$, so being of length $\mu(\partial T)=\mu(\bar{A})=c_{2}(A)$. Denote by $|\cdot|$ the area measure. Then,

$$
|R|=c_{2}(A)=I^{*} \mu(\omega)=\mathcal{E}_{2}(\mu)=\sum_{\alpha \in E} \mu\left(\partial T_{\alpha}\right)^{2}=\sum_{\alpha \in E}\left|Q_{\alpha}\right|=\left|\bigcup_{\alpha} Q_{\alpha}\right| .
$$

It is then enough to show that the family of squares is contained in $R$ to prove that it is a tiling. It is clear that all the family $\left\{Q_{\alpha}\right\}$ lies in between the two vertical sides of $R$, and that the horizontal room is fully filled, by additivity of the measure. Moreover, as already observed in the proof of part (ii) of Theorem 1, for every $\xi \in \partial T$ it must be

$$
1 \geq V_{2} \mu(\xi)=\sum_{\alpha<\xi} \mu\left(\partial T_{\beta}\right)=\sum_{\alpha<\xi} \ell(\alpha) .
$$

It follows that $\bigcup_{\alpha} Q_{\alpha} \subseteq R$ and $\left\{Q_{\alpha}\right\}_{\alpha}$ is a tiling.

(ii) Let the rectangle $R$ be tiled according to the combinatorics of a tree $T$, as described above. Then for each $\alpha \in E$, we have:

$$
\sum_{\beta \geq \alpha}|Q(\beta)|=\ell(\alpha)\left(1-\sum_{\beta<\alpha} \ell(\beta)\right) .
$$

Hence, it is immediate that if we define a measure on $\partial T$ by $\mu\left(\partial T_{\alpha}\right)=\ell(\alpha)$, it solves equation (4). Then the harmonic function $g=V_{2} \mu$ is bounded by 1 on $V$ and solves (1). By Theorem $1, \mu$ must be the equilibrium measure of some $F_{\sigma}$ subset $A$ of $\partial T$.

It might be interesting to informally discuss some features of the tiling, and its relation to the set $A$. The example below can provide a useful illustration of what we are saying here in general terms. 
For each $\xi$ in $\partial T$, let $\alpha_{n}$ be the only edge of level $n$ belonging to the ray $\xi$, and choose a point $x_{n+1}$ in $Q_{\alpha_{n}}$. Then, it is immediate that $\lim _{n \rightarrow \infty} x_{n}=: \lambda(\xi)$ exists in $R$, and that it does not depend on the precise location of the $x_{n}$ 's in the corresponding squares. Let $\pi(\xi)$ be the orthogonal projection of $\lambda(\xi)$ onto the lower side of $R$, identified with $\left[0, c_{2}(A)\right]$.

Let $A \subseteq \partial T$ and $\mu^{A}$ its equilibrium measure. The following facts are easy to check:

(i) if we replace $T$ by its subtree obtained keeping only the edges $\alpha$ with $\mu^{A}\left(\partial T_{\alpha}\right)>0$, then tiling does not have degenerate squares, and $c_{2}\left(A \cap \partial T_{\alpha}\right)>0$ for all edges $\alpha$.

(ii) let $A^{\prime}$ be the set of regular points for $\mu^{A}$, i.e. $A^{\prime}=\left\{\xi \in \partial T: V_{2} \mu^{A}(\xi)=1\right\}$ : then $\mu^{A^{\prime}}=\mu^{A}$, hence they induce the same tiling.

(iii) $\pi$ is injective but possibly at countably many points and surjective from $\partial T$ onto $\left[0, c_{2}(A)\right]$

(iv) $\mu^{A}\left(\pi^{-1}(A)\right)=\ell(A)$ for all measurable sets $A \subseteq\left[0, c_{2}(E)\right]$ (where $\ell$ denotes length measure on $\left.\left[0, c_{2}(A)\right]\right)$;

(v) let $\operatorname{Ex}(A):=\{\xi \in \partial T: \pi(\xi) \neq \lambda(\xi)\}$ : then, $\operatorname{Ex}(A)=\partial T \backslash A^{\prime}$;

(Fig. 1)

We can assume from now that the tree has been pruned as in (i) and that $A=A^{\prime}$. Still, we see below that the combinatorics of the tree are not, by themselves, enough to determine a rectangle $R$ and a square tiling of it. They are, if we assume that the set $A$ in the Theorem 2 is closed, but they are not in general. This is in striking contrast with the case of finite trees, or more generally graphs. However, if $c_{2}(A)<c_{2}(\partial T)$, then a price has to be paid. In fact, in that case

$$
c_{2}(\operatorname{Ex}(A)) \geq c_{2}(\partial T)-c_{2}(A)>0,
$$

Fig. 1 For the boundary point $\xi$ identified by the geodesic $\left\{x_{j}\right\}_{j}$, $\lambda(\xi)$ does not lie on the bottom side of $R$

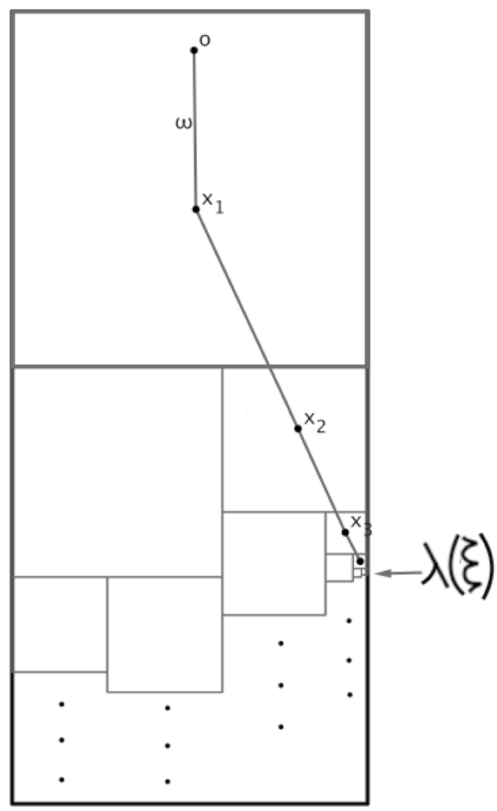


i.e., the exceptional set $\operatorname{Ex}(A)$ is rather large, although, clearly, $0=\mu^{A}(\operatorname{Ex}(A))=\ell(\pi(\operatorname{Ex}(A)))$. To enlighten this phenomenon, we provide an example of a set which is densly spread out on the boundary having exceptional set of full capacity.

Example 1 (A regular set of dyadic combinatorics and arbitrarily small capacity with positive capacity in every subtree) Let $\varepsilon>0$ be any small number, and $T=T_{2}$ a dyadic tree with edge root $\omega$. Let $n=n(\omega)$ be the number of steps one has to move to the left, starting from the root, before finding an edge $\alpha_{n}^{\omega}$ such that $c_{p}\left(\partial T_{n, \omega}\right) \leq \varepsilon / 2$, where $T_{n, \omega}$ denotes $\alpha_{n}^{\omega}-$ tent. Let $\left\{\alpha_{1}^{\omega}, \ldots, \alpha_{n}^{\omega}\right\}$ be the geodesic from the root to $\alpha_{n}^{\omega}$, and $\beta_{j}$ be the right brother of $\alpha_{j}^{\omega}$, i.e. the only edge with $b\left(\beta_{j}\right)=b\left(\alpha_{j}^{\omega}\right)$. In each subtree $T_{\beta_{j}}, j=1, \ldots, n$, starting from the root $\beta_{j}$, move to the left, say $n\left(\beta_{j}\right)$ steps, until you find an edge $\alpha_{n\left(\beta_{j}\right)}^{\beta_{j}}$ such that $c_{p}\left(\partial T_{n\left(\beta_{j}\right), \beta_{j}}\right) \leq \varepsilon /\left(2^{2} n\right)$. Then we iterate the process: $\left\{\alpha_{1}^{\beta_{j}}, \ldots, \alpha_{n\left(\beta_{j}\right)}^{\beta_{j}}\right\}$ is the geodesic from $\beta_{j}$ to $\alpha_{n\left(\beta_{j}\right)}^{\beta_{j}}$ and $\gamma_{i}=\gamma_{i}(j)$ the right brother of $\alpha_{i}^{\beta_{j}}$. In each subtree $T_{\gamma_{i}}$ we individuate as before, always moving to the left, subtrees with $c_{p}\left(\partial T_{n\left(\gamma_{i}\right), \gamma_{i}}\right) \leq \varepsilon /\left(2^{3} n\left(\beta_{j}\right)\right)$, and so on. Let

$$
A_{1}=\partial T_{\alpha_{n}^{\omega}}, \quad A_{2}=\bigcup_{j=1}^{n} \partial T_{n\left(\beta_{j}\right), \beta_{j}}, \quad A_{3}=\bigcup_{j=1}^{n} \bigcup_{i=1}^{n\left(\beta_{j}\right)} \partial T_{n\left(\gamma_{i}\right) \gamma_{i}}, \quad \ldots, \quad \text { and set } \quad A=\bigcup_{k} A_{k} .
$$

By construction, for every $\alpha \in E$ the tree $T_{\alpha}$ contains a tent with boundary in $A$. Since tents have positive capacity (for example by the rescaling property of Proposition 3 ), it follows that $c_{p}\left(A \cap \partial T_{\alpha}\right)>0$ for every $\alpha \in E$. On the other hand,

$$
c_{p}(A) \leq \sum_{k} c_{p}\left(A_{k}\right) \leq \sum_{k} \frac{\varepsilon}{2^{k}}=\varepsilon .
$$

For the regularity, observe that by construction for every point $\xi \in A$, there exists some edge $\alpha$ such that $\xi \in \partial T_{\alpha} \subseteq A$. Therefore, if $\mu, \mu^{\alpha}$ are the equilibrium measures for $A$ and $\partial T_{\alpha}$ respectively, we have

$$
V_{p} \mu(\xi)=V_{p, \alpha} \mu(\xi)+V_{p} \mu(b(\alpha))=\left(1-V_{p} \mu(b(\alpha))\right) V_{p, \alpha} \mu^{\alpha}(\xi)+V_{p} \mu(b(\alpha))=1,
$$

by the regularity of homogeneous trees.

\section{Branched continued fractions}

Theorem 1 can be reformulated in terms of branched continued fractions. Besides adding further interesting structure to the class of equilibrium measures, this provides a recursive formula for concretely calculating capacity of sets. An accessible survey on branched continued fractions is in [5].

Proposition 6 Let $f: E \rightarrow \mathbb{R}^{+}$be any non-negative function such that $I_{p} f<1$ on $V$, and consider the associated rescaled function defined by 


$$
c(\alpha)=\frac{f(\alpha)}{\left(1-I_{p} f(b(\alpha))\right)^{p-1}} .
$$

Then, $f$ is the potential of a measure $\mu$ on $\partial T$ if and only if $c$ is defined, for each edge $\alpha$ which is not a leaf, by the following recursive formula

$$
c(\alpha)=\frac{\sum_{\beta \in s(\alpha)} c(\beta)}{\left(1+\left(\sum_{\beta \in s(\alpha)} c(\beta)\right)^{p^{\prime}-1}\right)^{p-1}} .
$$

Proof By (10), $f(\omega)=c(\omega)$. Denote by $\alpha^{-}$the parent of $\alpha$, i.e. the only edge $\alpha^{-}$such that $\alpha \in s\left(\alpha^{-}\right)$. For every $\alpha \neq \omega$, we have

$$
\begin{aligned}
f(\alpha)^{p^{\prime}-1} & =c(\alpha)^{p^{\prime}-1}\left(1-I_{p} f(b(\alpha))\right)=c(\alpha)^{p^{\prime}-1}\left(1-I_{p} f\left(b\left(\alpha^{-}\right)\right)-f\left(\alpha^{-}\right)^{p^{\prime}-1}\right) \\
& =c(\alpha)^{p^{\prime}-1}\left(\frac{f\left(\alpha^{-}\right)^{p^{\prime}-1}}{c\left(\alpha^{-}\right)^{p^{\prime}-1}}-f\left(\alpha^{-}\right)^{p^{\prime}-1}\right)=c(\alpha)^{p^{\prime}-1} f\left(\alpha^{-}\right)^{p^{\prime}-1} \frac{1-c\left(\alpha^{-}\right)^{p^{\prime}-1}}{c\left(\alpha^{-}\right)^{p^{\prime}-1}} .
\end{aligned}
$$

Iterating we obtain,

$$
f(\alpha)=c(\alpha) \prod_{\gamma<\alpha}\left(1-c(\gamma)^{p^{\prime}-1}\right)^{p-1}
$$

Hence, for any chosen edge $\alpha$ which is not a leaf, it holds

$$
\sum_{\beta \in s(\alpha)} f(\beta)=\prod_{\gamma \leq \alpha}\left(1-c(\gamma)^{p^{\prime}-1}\right)^{p-1} \sum_{\beta \in s(\alpha)} c(\beta)
$$

Now, $f$ is the co-potential of a measure if and only if the flow condition (6) holds. Namely, using (12), (6) holds if and only if

$$
c(\alpha)=\left(1-c(\alpha)^{p^{\prime}-1}\right)^{p-1} \sum_{\beta \in s(\alpha)} c(\beta),
$$

which is equivalent to (11), as can be seen solving with respect to $c(\alpha)$.

By the rescaling properties of equilibrium measure (Proposition 3), we have that if $\mu$ is the $p$-equilibrium measure for a set $A \subseteq \partial T$, then $c(\alpha)=c_{p, \alpha}\left(A_{\alpha}\right)$. This gives us an algorithm to calculate the capacity of a set in $\partial T$ in terms of successive tents capacities. Moreover, by relation (11) we deduce that capacities can be expressed by means of branched continued fractions. For example, by (11) we obtain the expression

$$
c_{2}(\partial T)=\frac{1}{1+\frac{1}{\sum_{\beta \in s(\omega)} \frac{1}{1+\sum_{\gamma \in s(\beta)} \frac{1}{1+\ldots}}}}
$$


In [15, p. 57] the same structure was observed for the total resistence $R$ of an infinite tree without edges of degree 1 . In particular, for such a class of trees, we obtain the relation

$$
c_{2}(\partial T)=\frac{1}{1+R} .
$$

To end the section, we give a reformulation of Theorem 1 which provides a characterization of equilibrium measures by means of an equation for capacities.

Theorem 3 (i) Let $\mu$ the equilibrium measure for a set $A \subseteq \partial T$ and $f=I^{*} \mu$. Then the function $c=c_{f}$ given by $(10)$ solves

$$
c(\alpha)\left(1-c(\alpha)^{p^{\prime}-1}\right)=\sum_{\beta>\alpha} c(\beta)^{p^{\prime}} \prod_{\alpha \leq \gamma<\beta}\left(1-c(\gamma)^{p^{\prime}-1}\right)^{p}, \quad \alpha \in E .
$$

(ii) Let $\mu$ be a measure on $\partial T$ such that $V_{p}(\mu)<1$ on $V$. Set $f=I^{*} \mu$. If the function $c=c_{f}$ solves (13), then there exists an $\mathcal{F}_{\sigma}$ set $A \subseteq \partial T$ such that $\mu$ is its p-equilibrium measure.

Proof Given any measure $\mu$, setting $f=I^{*} \mu$ and $c=c_{f}$, by (10) we have

$$
f(\alpha)\left(1-I_{p} f(b(\alpha))\right)=\frac{f(\alpha)^{p^{\prime}}}{c(\alpha)^{p^{\prime}-1}}, \quad \alpha \in E .
$$

If $\mu$ is an equilibrium measure then (4) holds, and conversely if $\mu$ is such that $V_{p}(\mu)<1$ and solves (4) it is the equilibrium measure of some $\mathcal{F}_{\sigma}$ set. But (4) holds if and only if

$$
f(\alpha)^{p^{\prime}}\left(1-c(\alpha)^{p^{\prime}-1}\right)=c(\alpha)^{p^{\prime}-1} \sum_{\beta>\alpha} f(\beta)^{p^{\prime}}
$$

But by Proposition 6, we know that $c(\alpha)$ solves (11), or equivalently, $f(\alpha)$ is defined by (12). Hence, sobstituting above we get

$$
c(\alpha)^{p^{\prime}} \prod_{\gamma<\alpha}\left(1-c(\gamma)^{p^{\prime}-1}\right)^{p}\left(1-c(\alpha)^{p^{\prime}-1}\right)=c(\alpha)^{p^{\prime}-1} \sum_{\beta>\alpha} c(\beta)^{p^{\prime}} \prod_{\gamma<\beta}\left(1-c(\gamma)^{p^{\prime}-1}\right)^{p},
$$

which is (13).

Acknowledgements It is a pleasure to acknowledge useful discussions with Davide Cordella and Nikolaos Chalmoukis.

Funding Open access funding provided by Politecnico di Torino within the CRUI-CARE Agreement.

Open Access This article is licensed under a Creative Commons Attribution 4.0 International License, which permits use, sharing, adaptation, distribution and reproduction in any medium or format, as long as you give appropriate credit to the original author(s) and the source, provide a link to the Creative Commons licence, and indicate if changes were made. The images or other third party material in this article are included in the article's Creative Commons licence, unless indicated otherwise in a credit line to the material. If material is not included in the article's Creative Commons licence and your intended use is not permitted by statutory regulation or exceeds the permitted use, you will need to obtain permission directly from the copyright holder. To view a copy of this licence, visit http://creativecommons.org/licenses/by/4.0/. 


\section{References}

1. Adams, D.R., Hedberg, L.I.: Function Spaces and Potential Theory, Vol. 314. Springer (2012)

2. Arcozzi, N., Rochberg, R., Sawyer, E.: Capacity, carleson measures, boundary convergence, and exceptional sets. Perspectives in partial differential equations, harmonic analysis and applications. Proc. Sympos. Pure Math 79, 1-20 (2008)

3. Arcozzi, N., Rochberg, R., Sawyer, E.T., Wick, B.D.: Potential theory on trees, graphs and ahlfors-regular metric spaces. Potential Anal. 41(2), 317-366 (2014)

4. Benjamini, I., Schramm, O.: Random walks and harmonic functions on infinite planar graphs using square tilings. Ann. Prob. 24(3), 1219-1238 (1996)

5. Bodnar, D.: On the convergence of branched continued fractions. J. Math. Sci. 97(1), 3862-3871 (1999)

6. Brelot, M., Gowrisankaran, K., Murthy, M.V.: Lectures on potential theory, vol. 19. Tata Institute of Fundamental Research Bombay (1967)

7. Brooks, R.L., Smith, C.A.B., Stone, A.H., Tutte, W.T., et al.: The dissection of rectangles into squares. Duke Math. J. 7(1), 312-340 (1940)

8. Chalmoukis, N., Levi, M.: Some remarks on the dirichlet problem on infinite trees. Concret. Oper. 6(1), 20-32 (2019)

9. Choquet, G.: Theory of capacities. In: Annales de l'institut Fourier, vol. 5, pp. 131-295 (1954)

10. Chung, K.L.: Probabilistic approach in potential theory to the equilibrium problem. In: Annales de l'institut Fourier, vol. 23, pp. 313-322 (1973)

11. Chung, K.L., Rao, M.: Equilibrium and energy. In: Selected Works Of Kai Lai Chung, pp. 631-640. World Scientific (2008)

12. Georgakopoulos, A.: The boundary of a square tiling of a graph coincides with the poisson boundary. Invent. Math. 203(3), 773-821 (2016)

13. Lyons, R., Peres, Y.: Probability on trees and networks, vol. 42. Cambridge University Press (2017)

14. Schramm, O.: Square tilings with prescribed combinatorics. Israel J. Math. 84(1-2), 97-118 (1993)

15. Soardi, P.M.: Potential Theory on Infinite Networks. Springer (2006)

16. Woess, W.: Denumerable markov chains. generating functions, boundary theory, random walks on trees ems textbk. Math. Eur. Math. Soc., Zürich Crossref MathSciNet ZentralBlatt Math (2009)

Publisher's Note Springer Nature remains neutral with regard to jurisdictional claims in published maps and institutional affiliations. 